\section{A Meningioma Exclusively Located inside the Superior Sagittal Sinus Responsible for Intracranial Hypertension}

We report a case of a 57-year-old patient with a history of intracranial hypertension (visual impairment, headaches, and papillary edema) due to a meningioma exclusively located inside the superior sagittal sinus (SSS). The lesion was not detected on a nonenhanced brain CT. MR imaging revealed a centimetric round homogeneous lesion, hyperintense on T2-weighted images, intensely and homogeneously enhanced after gadolinium injection on T1-weighted sequences with fat suppression (Fig 1A), inside the distal part of the SSS. On venous MR angiography, the lesion appeared as a filling defect inside the SSS (Fig $1 B$ ). The tumor did not grow (1-year interval between the $2 \mathrm{MR}$ imaging examinations), and its characteristics on MR imaging led to the diagnosis of stage I meningioma.

We performed an angiographic examination: The arterial phase revealed a tumor blush originating from the left middle cerebral artery, highly evocative of the hypervascularization of a meningioma
(Fig $2 A$ ), and the venous phase showed an SSS stenosis correlating with the tumor blush (Fig $2 B$ ). The patient was treated with acetazolamide (Diamox), but after a year, headaches worsened and an angiographic treatment was required. A stent was inserted through a catheter directed into the SSS from a percutaneous jugular puncture. The patient was heparinized during the procedure and then given aspirin for 6 months. Clinical relief was achieved within 4 months. No histologic proof could be obtained, but the patient was referred to another hospital for stereotactic radiosurgery to treat the meningioma.

Meningiomas account for $13 \%-26 \%$ of all intracranial tumors and represent the most common nonglial primary tumors of the brain. ${ }^{1}$ The incidence is approximately 6 per 100,000 and is higher among the elderly. They are composed of neoplastic meningothelial cells originating from the arachnoid layer of the meninges. Atypical development sites are the ventricular system, the orbit, the scalp, the paranasal sinus, the nasopharynx, the neck, and the skin. In our patient, the lesion was exclusively located inside the SSS. To our knowl-
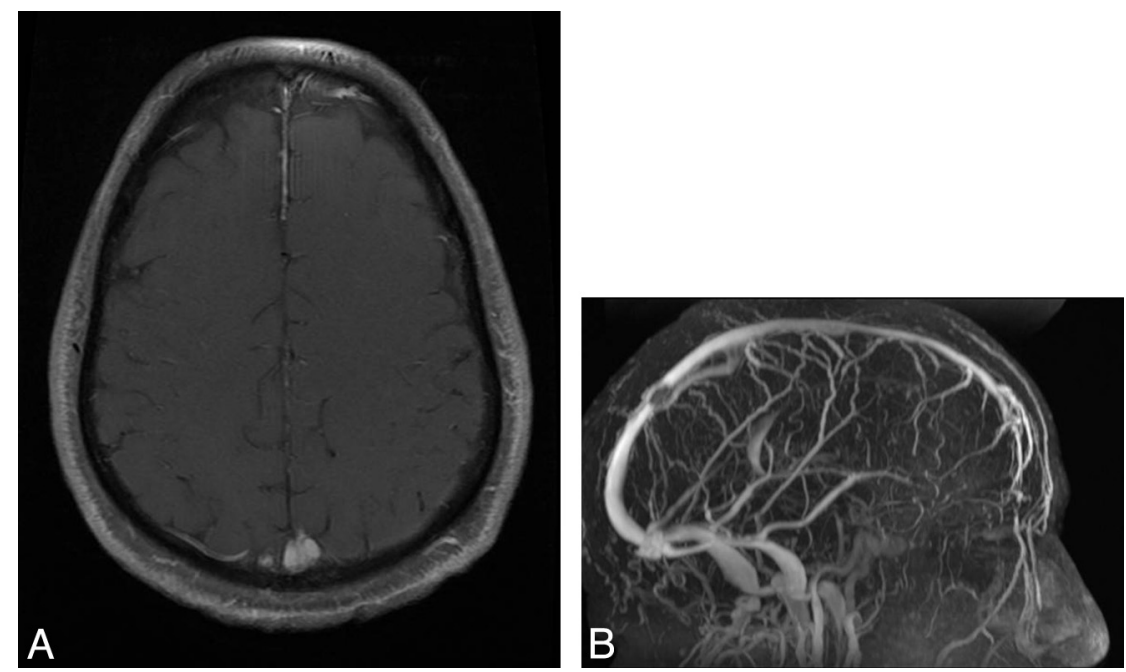

Fig 1. $A$, The T1-weighted sequence after gadolinium injection and fat suppression reveals an endovascular mass, intensely and homogeneously enhanced. The tumor's characteristics on all the sequences are consistent with meningioma. $B$, The MR venography shows a filling defect inside the SSS corresponding to an endoluminal tumor.
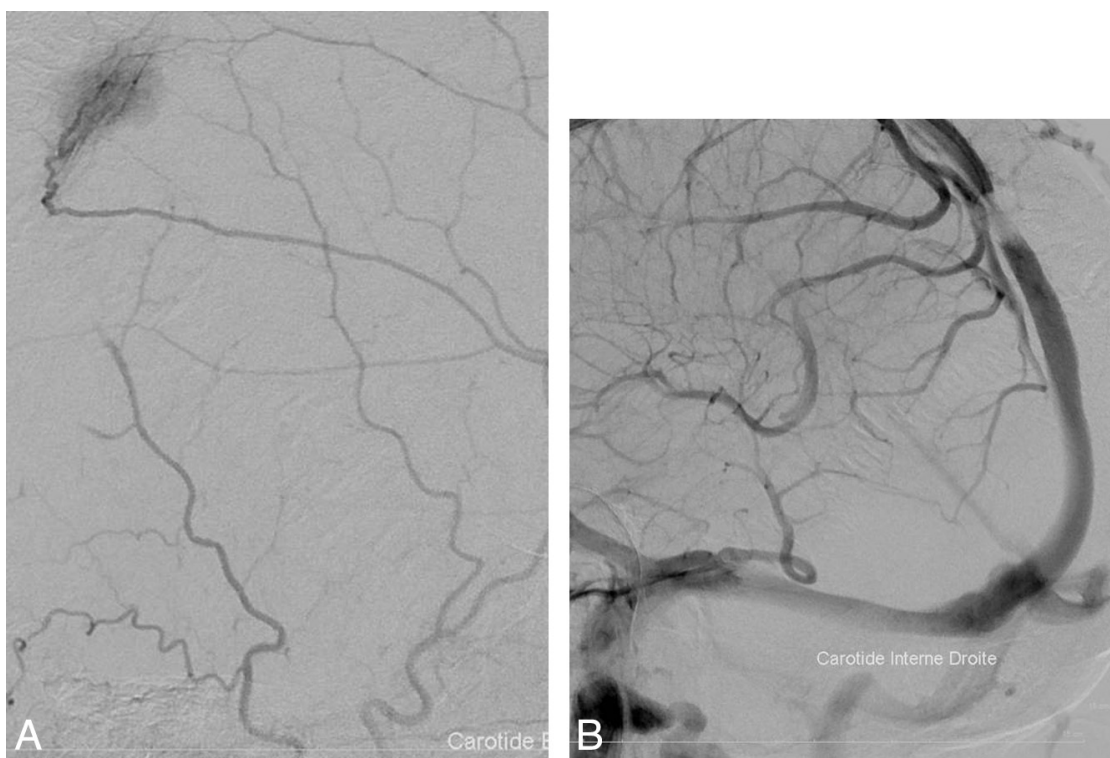

Fig 2. A, Angiographic exam: the arterial phase reveals a tumor blush typical of meningiomas' hypervascularization. The arterioles come from the left middle cerebral artery. $B$, The venous phase shows a SSS stenosis. 
edge, no such location has been reported so far, though intravenous extension of intracranial meningiomas occurs in approximately $20 \%$ of cases. $^{2}$

There are several therapeutic options for treating meningiomas: Surgery can be used in $80 \%$ of cases. ${ }^{3}$ Radiation therapy is used in case of tumoral progression or incomplete resection, and radiosurgery has become the treatment of choice for lesions $<3 \mathrm{~cm}$ and/or inaccessible to surgery.

The close correlation between CSF pressure and venous pressure in the SSS explains how a venous sinus obstruction causes intracranial hypertension. It was proved by simultaneous CSF and venous manometry ${ }^{4}$ that a venous pressure rise led to a CSF absorption dysfunction and, consequently, higher CSF volume and pressure. Benign intracranial hypertension can be classified into 2 categories depending on the underlying etiology ${ }^{4}$ : the idiopathic type (also called pseudotumor cerebri) and the venogenic type when there is a primary pathologic condition of the venous sinus (thrombosis, vasculitis, extrinsic benign compression, or intraluminal tumor as in our patient).

Venous stent placement is a safe and effective procedure to relieve dural sinus obstructions. ${ }^{4}$ Full-dose heparin should be administered for 24-48 hours after the procedure; then, the heparin should be replaced by an antiplatelet agent until the stent is coated by the vascular epithelium (6 months).

\section{References}

1. O'Leary S, Adams WM, Parrish RW, et al. Atypical imaging appearances of intracranial meningiomas. Clin Radiol 2007;62:10-17

2. Vachhrajani S, Jea A, Rutka JA, et al. Meningioma with dural venous sinus invasion and jugular vein extension. J Neurosurg Pediatr 2008;2:391-96

3. Marosi C, Hassler M, Roessler K, et al. Meningioma. Crit Rev Oncol Hematol 2008;67:153-71

4. Owler B, Parker G, Halmagyi GM, et al. Pseudotumor cerebri syndrome: venous sinus obstruction and its treatment with stent placement. J Neurosurg 2003;98:1045-55

B. Szitkar

Department of Radiology

Amiens Teaching Hospital

Salouel, France

DOI 10.3174/ajnr.A2130 